\title{
Signatures of Dark Matter Scattering Inelastically Off Nuclei
}

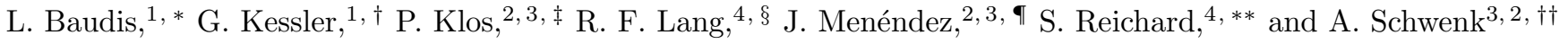 \\ ${ }^{1}$ Physics Institute, University of Zürich, Winterthurerstr. 190, CH-805\%, Switzerland \\ ${ }^{2}$ Institut für Kernphysik, Technische Universität Darmstadt, D-64289 Darmstadt, Germany \\ ${ }^{3}$ ExtreMe Matter Institute EMMI, GSI Helmholtzzentrum für Schwerionenforschung GmbH, D-64291 Darmstadt, Germany \\ ${ }^{4}$ Department of Physics, Purdue University, West Lafayette, IN 4790\%, USA
}

\begin{abstract}
Direct dark matter detection focuses on elastic scattering of dark matter particles off nuclei. In this study, we explore inelastic scattering where the nucleus is excited to a low-lying state of $10-100 \mathrm{keV}$, with subsequent prompt de-excitation. We calculate the inelastic structure factors for the odd-mass xenon isotopes based on state-of-the-art large-scale shell-model calculations with chiral effective field theory WIMP-nucleon currents. For these cases, we find that the inelastic channel is comparable to or can dominate the elastic channel for momentum transfers around $150 \mathrm{MeV}$. We calculate the inelastic recoil spectra in the standard halo model, compare these to the elastic case, and discuss the expected signatures in a xenon detector, along with implications for existing and future experiments. The combined information from elastic and inelastic scattering will allow for the determination of the dominant interaction channel within one experiment. In addition, the two channels probe different regions of the dark matter velocity distribution and can provide insight into the dark halo structure. The allowed recoil energy domain and the recoil energy at which the integrated inelastic rates start to dominate the elastic channel depend on the mass of the dark matter particle, thus providing a potential handle to constrain its mass.
\end{abstract}

PACS numbers: 95.35.+d, 14.80.Ly, 21.60.Cs, 29.40.-n

Keywords: Inelastic scattering, dark matter, direct detection, xenon

\section{INTRODUCTION}

Astrophysical evidence indicates that the milky way disc is embedded into a non-baryonic dark matter halo [1], which could be made of weakly interacting massive particles (WIMPs) 2]. The WIMP dark matter hypothesis is testable, as WIMPs may be detected directly by scattering off nuclei in low-background underground detectors, indirectly by observing annihilation products above astrophysical backgrounds, and by producing them at the LHC [3]. In direct detection experiments, only the elastic scattering channel, with an exponential nuclear recoil energy spectrum, is usually exploited 4 .

Another avenue to direct detection is to observe inelastic WIMP-nucleus scattering, inducing transitions to low-lying excited states [5]. The experimental signature is a nuclear recoil together with the prompt de-excitation photon. In odd-mass nuclei with low-lying excited states, a galactic WIMP with sufficient kinetic energy can induce inelastic excitations. Xenon is an excellent target material because natural xenon contains the odd ${ }^{129} \mathrm{Xe}$ and ${ }^{131} \mathrm{Xe}$ isotopes with abundances of $26.4 \%$ and $21.2 \%$, respectively. The excitation energies of the lowest-lying states are reasonably low, with a $3 / 2^{+}$state at $39.6 \mathrm{keV}$ above the $1 / 2^{+}$ground state in ${ }^{129} \mathrm{Xe}$ and a $1 / 2^{+}$state

\footnotetext{
* laura.baudis@physik.uzh.ch

$\dagger$ gaudenz.kessler@physik.uzh.ch

$\ddagger$ pklos@theorie.ikp.physik.tu-darmstadt.de

$\S \frac{\text { rafael@purdue.edu }}{\text { ravier.menendez@phy }}$

ๆ javier.menendez@physik.tu-darmstadt.de

** sreichar@purdue.edu

†† schwenk@physik.tu-darmstadt.de
}

at $80.2 \mathrm{keV}$ above the $3 / 2^{+}$ground state in ${ }^{131} \mathrm{Xe}$. The nuclear decays are electromagnetic M1 and E2 with halflives of $0.97 \mathrm{~ns}$ and $0.48 \mathrm{~ns}$, respectively. Searches for inelastic scattering have been performed in the past [6] 9].

Here, we show that inelastic WIMP-nucleus scattering in xenon is complementary to elastic scattering for spindependent interactions, as the inelastic channel dominates the integrated spectra above $\sim 10 \mathrm{keV}$ energy deposition, depending on the WIMP mass. This aspect not only provides a consistency check for a xenon experiment, but in the case of dark matter detection via this channel, it would offer a clear indication for the spin-dependent nature of the fundamental interaction.

This paper is organized as follows. In Sect. II we calculate the structure factors for inelastic WIMP-nucleus scattering, which is dominated by spin-dependent interactions for the relevant low-lying excited states. The kinematics of inelastic scattering is described in Sect. III. In Sect. IV, we explore the expected inelastic signature in a xenon dark matter detector and compare it with the signal region for elastic nuclear recoils. Finally, we discuss in Sect. V] the implications of this signature for current and future dark matter detectors such as LUX, LZ, XENON, XMASS, and DARWIN, and conclude.

\section{INELASTIC STRUCTURE FACTORS}

\section{A. Nuclear structure and WIMP-nucleon currents}

The calculation of inelastic WIMP scattering off nuclei requires a reliable description of the structure of the initial and final nuclear states as well as the WIMP-nucleon 
currents. Because low-lying transitions occur in oddmass isotopes with different spins of the ground and excited states, spin-dependent WIMP scattering generally dominates for the inelastic channel (see Ref. [10, which studied the spin-independent case). We therefore consider only spin-dependent WIMP-nucleon interactions.

We perform state-of-the-art large-scale nuclear structure calculations for ${ }^{129} \mathrm{Xe}$ and ${ }^{131} \mathrm{Xe}$ using the shellmodel code ANTOINE 11] and the GCN5082 interaction [12, 13] in the $0 g_{7 / 2}, 1 d_{5 / 2}, 1 d_{3 / 2}, 2 s_{1 / 2}$, and $0 h_{11 / 2}$ valence space on top of a ${ }^{100} \mathrm{Sn}$ core as in Refs. 14, 15. For ${ }^{129} \mathrm{Xe}$, the number of particle excitations into the last three orbitals was limited to three; for ${ }^{131} \mathrm{Xe}$, we perform an exact diagonalization. These present the largest valence spaces with nuclear interactions that have been tested in nuclear structure and decay studies. The resulting energy spectra show a very good overall agreement with experimental data [14, and the spins of the ground and first excited states are correctly predicted, a clear improvement with respect to previous work [16]. The calculated excitation energies for the first excited states in ${ }^{129} \mathrm{Xe}$ and ${ }^{131} \mathrm{Xe}, 107 \mathrm{keV}$ and $37 \mathrm{keV}$, are in reasonable agreement with the experimental values, given these very small energies. Note that for the inelastic WIMPnucleus cross sections, we will use the experimental excitation energies, so the calculation uses as input only the wave functions of the nuclear states.

While WIMPs interact with quarks, at the momentum scales relevant to nuclei and WIMP-nucleus scattering, the relevant degrees of freedom are nucleons and pions. In this regime, chiral effective field theory (EFT) provides a systematic expansion in powers of momentum $Q$ for the coupling of WIMPs to nucleons based on the symmetries of QCD. At leading orders $Q^{0}$ and $Q^{2}$, chiral EFT predicts one-body (1b) currents given by [14, 15]

$$
\begin{aligned}
\sum_{i=1}^{A} \mathbf{J}_{i, 1 \mathrm{~b}}^{3}= & \sum_{i=1}^{A} \frac{1}{2}\left[a_{0} \boldsymbol{\sigma}_{i}\right. \\
& \left.+a_{1} \tau_{i}^{3}\left(\frac{g_{A}\left(p^{2}\right)}{g_{A}} \boldsymbol{\sigma}_{i}-\frac{g_{P}\left(p^{2}\right)}{2 m g_{A}}\left(\mathbf{p} \cdot \boldsymbol{\sigma}_{i}\right) \mathbf{p}\right)\right],
\end{aligned}
$$

where the sum is over all $A$ nucleons in the nucleus, and we consider spin- $1 / 2$ WIMPs. These $1 \mathrm{~b}$ currents are similar to the phenomenological currents that have been used in spin-dependent WIMP scattering off nuclei [17. Here, $a_{0}$ and $a_{1}$ are the isoscalar and isovector WIMP-nucleon couplings; $\boldsymbol{\sigma}_{i}$ and $\tau_{i}^{3}$ are spin and isospin matrices; and $\mathbf{p}=\mathbf{p}_{i}-\mathbf{p}_{f}$ denotes the momentum transfer from nucleons to WIMPs. $g_{A}\left(p^{2}\right)$ and $g_{P}\left(p^{2}\right)$ are the axial and pseudoscalar couplings including $Q^{2}$ corrections given in Ref. 14], $g_{A}(0)=g_{A}$ and $m$ is the nucleon mass.

In addition to the coupling through $1 \mathrm{~b}$ currents, chiral EFT predicts two-body (2b) currents at order $Q^{3}$, where WIMPs couple to two nucleons [14]. Two-body currents are quantitatively important in medium-mass and heavy nuclei because the momentum $Q$ involved is not only sensitive to the momentum transfer but also to the typical momenta of nucleons in nuclei, which is higher (of order of the Fermi momentum) [18. The normal-ordered $1 \mathrm{~b}$ part of the dominant long-range $2 \mathrm{~b}$ currents is derived summing over occupied states in a spin-isospin symmetric reference state, which we take as a Fermi gas [14, 15, 18. This is expected to be a very good approximation due to phase-space considerations [19] and allows direct comparison to Eq. (1). The inclusion of the long-range $2 \mathrm{~b}$ currents leads to additional contributions to the isovector axial and pseudoscalar currents. Combining the $1 \mathrm{~b}$ and $2 \mathrm{~b}$ currents to order $Q^{3}$ gives [15]

$$
\begin{aligned}
\mathbf{J}_{i, 1 \mathrm{~b}+2 \mathrm{~b}}^{3}= & \frac{1}{2} a_{1} \tau_{i}^{3}\left[\left(\frac{g_{A}\left(p^{2}\right)}{g_{A}}+\delta a_{1}(p)\right) \boldsymbol{\sigma}_{i}\right. \\
& \left.+\left(-\frac{g_{P}\left(p^{2}\right)}{2 m g_{A}}+\frac{\delta a_{1}^{P}\left(p^{2}\right)}{p^{2}}\right)\left(\mathbf{p} \cdot \boldsymbol{\sigma}_{i}\right) \mathbf{p}\right],
\end{aligned}
$$

with a momentum- and density-dependent renormalization of the axial-vector and pseudoscalar parts, $\delta a_{1}(p)$ and $\delta a_{1}^{P}(p)$, respectively,

$$
\begin{aligned}
\delta a_{1}(p)= & -\frac{\rho}{F_{\pi}^{2}}\left[\frac{1}{3}\left(c_{4}+\frac{1}{4 m}\right)\left[3 I_{2}^{\sigma}(\rho, p)-I_{1}^{\sigma}(\rho, p)\right]\right. \\
& \left.+\frac{1}{3}\left(-c_{3}+\frac{1}{4 m}\right) I_{1}^{\sigma}(\rho, p)-\left(\frac{1+\hat{c}_{6}}{12 m}\right) I_{c 6}(\rho, p)\right],
\end{aligned}
$$

$$
\begin{aligned}
\delta a_{1}^{P}(p)= & \frac{\rho}{F_{\pi}^{2}}\left[\frac{-2 c_{3} p^{2}}{m_{\pi}^{2}+p^{2}}\right. \\
& \left.+\frac{c_{3}+c_{4}}{3} I^{P}(\rho, p)-\frac{1+\hat{c}_{6}}{12 m} I_{c 6}(\rho, p)\right],
\end{aligned}
$$

with density $\rho$, pion mass and decay constant $m_{\pi}, F_{\pi}$, and chiral EFT low-energy couplings $c_{3}, c_{4}, \hat{c}_{6}$. The values for the couplings and the functions $I_{1}^{\sigma}(\rho, p), I_{2}^{\sigma}(\rho, p)$, $I^{P}(\rho, p)$, and $I_{c 6}(\rho, p)$ (due to integrals in the exchange terms) are given and explained in detail in Ref. [15.

The uncertainties in chiral $2 \mathrm{~b}$ currents are dominated by the uncertainties of the $c_{3}$ and $c_{4}$ coupling. Considering a conservative range of values accepted in the literature leads to $\delta a_{1}(0)=-(0.14-0.32)$ [15] (the dependence on $p$ is very weak), so that $2 \mathrm{~b}$ currents reduce the axial part of the WIMP-nucleon currents. On the other hand, $\delta a_{1}^{P}\left(m_{\pi}\right)=0.23-0.54$ [15], leads to an enhancement of the pseudoscalar part. This enhancement vanishes at $p=0$, due to the pseudoscalar nature, but increases with momentum transfer.

\section{B. Structure factors}

Based on the initial $(i)$ and $(f)$ final states, we calculate the structure factors of inelastic, spin-dependent WIMP-nucleus scattering. The structure factor $S_{A}(p)$ receives contributions from longitudinal $\left(\mathcal{L}^{5}\right)$, transverse electric $\left(\mathcal{T}^{\text {el5 }}\right)$ and transverse magnetic $\left(\mathcal{T}^{\text {mag5 }}\right)$ multi- 
poles [15]:

$$
\begin{aligned}
S_{A}(p)= & \sum_{L \geqslant 0}\left|\left\langle J_{f}\left\|\mathcal{L}_{L}^{5}\right\| J_{i}\right\rangle\right|^{2} \\
& +\sum_{L \geqslant 1}\left(\left|\left\langle J_{f}|| \mathcal{T}_{L}^{\mathrm{el} 5}|| J_{i}\right\rangle\right|^{2}+\left|\left\langle J_{f} \| \mathcal{T}_{L}^{\operatorname{mag} 5}|| J_{i}\right\rangle\right|^{2}\right),
\end{aligned}
$$

where the detailed expressions for the multipoles in terms of the $1 b+2 b$ currents of Eq. (2) are given in Ref. 15] (see Eqs. (22)-(24)). Due to parity conservation, only odd/even $L$ multipoles contribute for the electric/magnetic multipoles when the inelastic transition is between nuclear states with the same parity (as is the case for xenon). While magnetic multipoles do not contribute to elastic scattering because of time-reversal symmetry, they do contribute to the inelastic case [15].

The inelastic structure factors for WIMP scattering off ${ }^{129} \mathrm{Xe}$ and ${ }^{131} \mathrm{Xe}$ are shown in Figure 1. Our results are presented in terms of the structure factors with "proton-only" $\left(a_{0}=a_{1}=1\right)$ and "neutron-only" $\left(a_{0}=-a_{1}=1\right)$ couplings, $S_{p}(u)$ and $S_{n}(u)$, as a function of the dimensionless momentum transfer $u=p^{2} b^{2} / 2$ with harmonic-oscillator length $b$. These coupling choices are more sensitive to protons and neutrons, respectively. Because xenon has an even number of protons, practically all the spin, and therefore the nuclear spin response, is dominated by neutrons, which also dominate the "proton-only" structure factors at low momentum transfer through the proton-neutron strong interaction in $2 \mathrm{~b}$ currents. For an extended discussion see Ref. [15. Data files of both the elastic 14, 15, and inelastic structure functions are available as Supplemental Material in the Appendix.

In Figure 2 we compare the inelastic structure factors to the elastic ones from Refs. [14, 15] for the experimentally relevant region. At $p=0$, both inelastic $S_{p}(u)$ and $S_{n}(u)$ are significantly smaller than their elastic counterparts, a factor 10 for ${ }^{129} \mathrm{Xe}$ and more than two orders of magnitude for ${ }^{131} \mathrm{Xe}$. However, for both isotopes, we observe a maximum in the inelastic structure factors at low momentum transfer, which was also found in previous calculations [16, 20]. This feature is absent in elastic scattering, where the maximum always occurs at $p=0$ and then sharply decreases 14, 15. As a result, for $u=1-2$ $(p=125-175 \mathrm{MeV})$, the inelastic channel is comparable to the elastic one. This is relevant because it is within the range of allowed momentum transfers in inelastic scattering (see Sect. III). In particular, for ${ }^{129} \mathrm{Xe}$, the inelastic structure factors even dominate their elastic counterparts. For ${ }^{131} \mathrm{Xe}$, the inelastic structure factors are slightly smaller but comparable to the elastic case.

Similar results comparing inelastic and elastic scattering were found in Ref. [16], although their structure factors are very different to ours. For example, in ${ }^{129} \mathrm{Xe}$, the inelastic structure factor is always decreasing in Ref. [16]; for ${ }^{131} \mathrm{Xe}$, the suppression of the inelastic response at $p=0$ is an order of magnitude smaller than in our

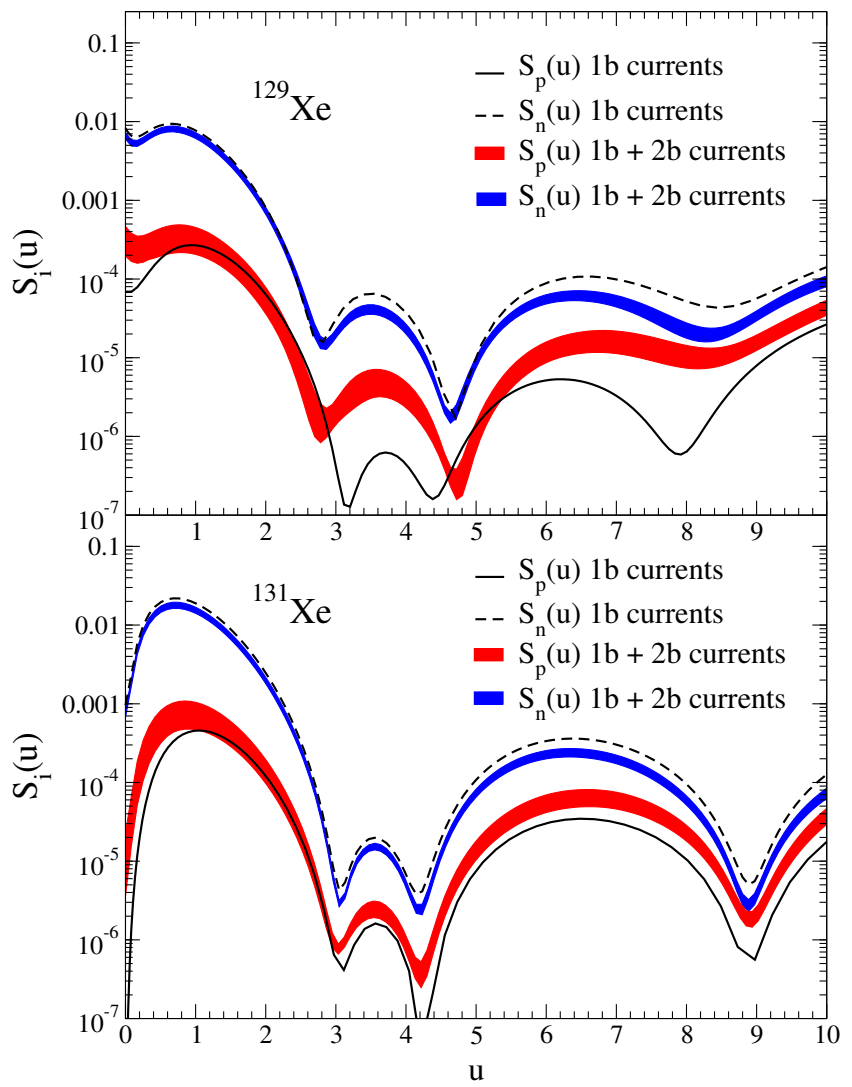

FIG. 1. (Color online) Inelastic structure factors $S_{p}$ (solid lines) and $S_{n}$ (dashed) for ${ }^{129} \mathrm{Xe}$ (top panel) and ${ }^{131} \mathrm{Xe}$ (bottom panel) as a function of $u=p^{2} b^{2} / 2$. The harmonicoscillator length is $b=2.2853 \mathrm{fm}$ for ${ }^{129} \mathrm{Xe}$ and $b=2.2905 \mathrm{fm}$ for ${ }^{131} \mathrm{Xe}$. Results are shown at the one-body (1b) current level and including two-body (2b) currents. The estimated theoretical uncertainty in the $2 \mathrm{~b}$ currents is given by the red $\left(S_{p}(u)\right)$ and blue $\left(S_{n}(u)\right)$ bands.

case, which is compensated by a rather flat momentumtransfer dependence; and both elastic and inelastic structure factors for ${ }^{131} \mathrm{Xe}$ in Ref. [16] are larger than ours at $u \sim 1$. These differences are due to the well-tested interactions and the smaller truncations in the present calculations (see also Ref. [14).

To understand the behavior at $p=0$ and the maxima in the inelastic structure factors compared to the elastic case, we have to consider the spin and parity of the ground state of ${ }^{129} \mathrm{Xe}, 1 / 2^{+}$, and its first excited state, $3 / 2^{+}$. The situation is reversed in ${ }^{131} \mathrm{Xe}$ with a $3 / 2^{+}$ ground state and a $1 / 2^{+}$first excited state. Therefore, only $L=1,2$ multipoles contribute to inelastic scattering. For elastic scattering, where the magnetic multipoles do not contribute, the allowed multipoles are $L=1$ for ${ }^{129} \mathrm{Xe}$ and $L=1,3$ for ${ }^{131} \mathrm{Xe}$. At $p=0$, only $L=1 \mathrm{mul}-$ tipoles contribute to the scattering, and only orbitals of the initial and final states with the same orbital angular momentum $l$ can be connected [15]. Because these contributions are naturally maximal in elastic scattering, but 


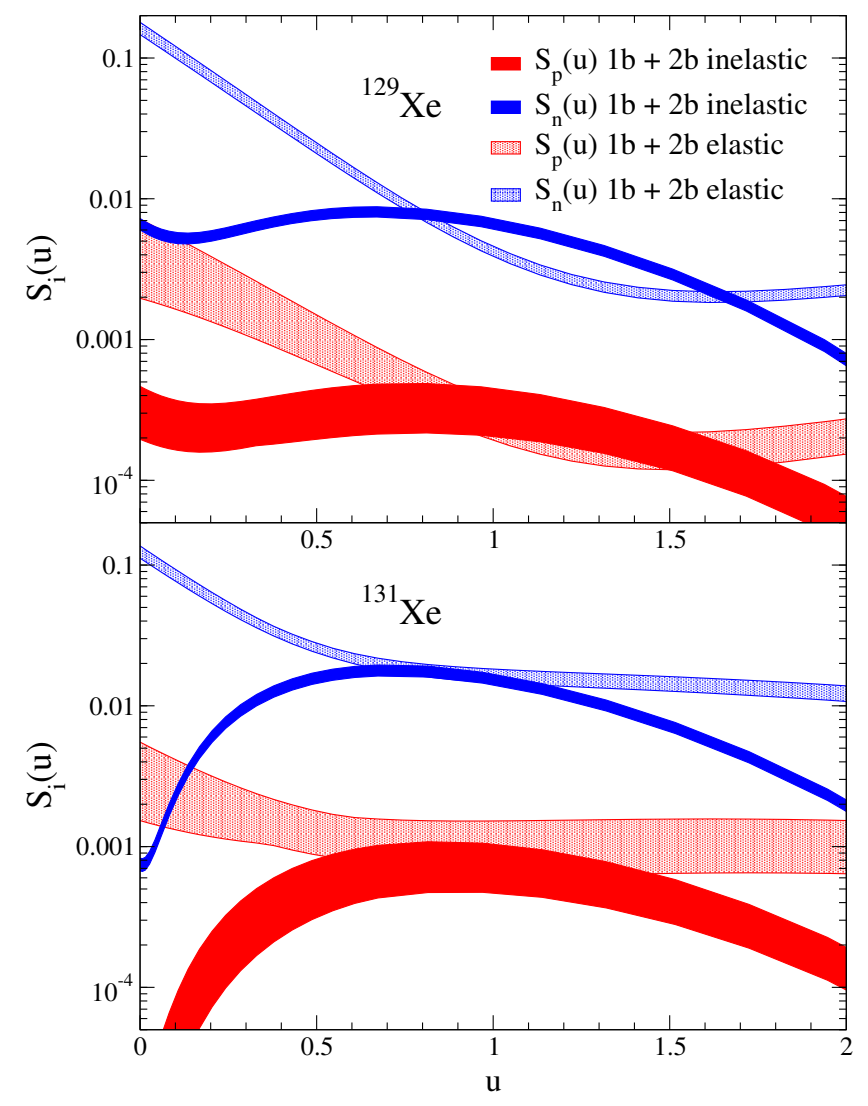

FIG. 2. (Color online) Comparison of the inelastic structure factors from Figure 1 to elastic counterparts from Ref. [14, 15]. Data files with these structure factors are available as supplementary material.

smaller when nucleons are excited into different $l$ orbitals, there is a strong reduction in the inelastic structure factors at $p=0$ compared to the elastic case.

At $p>0$, all multipoles contribute, and inelastic scattering is no longer suppressed, leading to a maximum in the structure factor. Moreover, because magnetic multipoles with $L=2$ only contribute to inelastic scattering, this response will be enhanced with respect to the elastic case. The extent of the suppression at $p=0$ and the maximum of the inelastic structure factor depend on the nuclear structure details of the states involved and on the relative contribution of the magnetic multipoles. For $p=0$, considering $1 \mathrm{~b}$ currents only, the inelastic structure factors can be related to the spin part of magnetic dipole $B(M 1)$ transitions. However, a precise fine-tuning to experimental $B(M 1)$ values probes different physics because, first, $2 \mathrm{~b}$ currents are different for electromagnetic and WIMP-nucleon interactions and, second, magnetic multipoles vanish at $p=0$, while they are crucial for the inelastic structure factors.

\section{KINEMATICS OF INELASTIC SCATTERING}

Given the promising results for the inelastic structure factors, we calculate the expected recoil spectra for inelastic dark matter scattering. The WIMP-nucleus scattering must conserve momentum and energy:

$$
\begin{aligned}
\mathbf{q}_{i} & =\mathbf{q}_{f}+\mathbf{q}, \\
\frac{q_{i}^{2}}{2 m_{\chi}} & =\frac{q_{f}^{2}}{2 m_{\chi}}+E_{R}+E^{*},
\end{aligned}
$$

where $\mathbf{q}_{i}$ and $\mathbf{q}_{f}$ are the initial and final WIMP momenta, $\mathbf{q}=\mathbf{q}_{i}-\mathbf{q}_{f}(=-\mathbf{p})$ is the momentum transfer, $m_{\chi}$ is the WIMP mass, and $E^{*}$ the excitation energy of the nucleus. The nuclear recoil energy is given by

$$
E_{R}=\frac{q^{2}}{2 m_{A}},
$$

with mass of the nucleus $m_{A}$. Eliminating $q_{f}$ in Eq. (7), this leads to a quadratic equation for $q$,

$$
q^{2}-\left(2 \mu v_{i} \cos \beta\right) q+2 \mu E^{*}=0
$$

with the reduced mass $\mu=m_{A} m_{\chi} /\left(m_{A}+m_{\chi}\right)$, initial WIMP velocity $v_{i}=q_{i} / m_{\chi}$, and where $\beta$ is the angle between $\mathbf{q}_{\mathbf{i}}$ and $\mathbf{q}$. This equation has two solutions:

$$
q_{ \pm}=\mu v_{i} \cos \beta\left(1 \pm \sqrt{1-\frac{2 E^{*}}{\mu v_{i}^{2} \cos ^{2} \beta}}\right) .
$$

This leads to two constraints:

$$
E^{*} \leqslant \frac{1}{2} \mu v_{i}^{2} \cos ^{2} \beta \leqslant \frac{1}{2} \mu v_{i}^{2},
$$

and

$$
v_{i}=\frac{1}{\cos \beta}\left(\frac{q}{2 \mu}+\frac{E^{*}}{q}\right) \geqslant \frac{q}{2 \mu}+\frac{E^{*}}{q}=v_{\min },
$$

where the allowed range is $\cos \beta \geqslant 0$. The upper limit in Eq. 111 on the excitation energy shows that the inelastic excitation is more sensitive to WIMPs with high velocities in the tail of the dark matter halo distribution. Because $q_{-}\left(q_{+}\right)$is monotonically decreasing (increasing) with respect to $v_{i} \cos \beta$, the absolute minimal (maximal) momentum transfer occurs at $v_{i} \cos \beta=v_{i}$ for $\cos \beta=1$. This defines the minimal and maximal recoil energies:

$$
E_{R, \min / \max }=\frac{\left(\mu v_{i}\right)^{2}}{2 m_{A}}\left(1 \mp \sqrt{1-\frac{2 E^{*}}{\mu v_{i}^{2}}}\right)^{2} .
$$

In the Earth's rest frame, the maximal $v_{i}$ is given by $v_{\text {esc }}+v_{\text {Earth }}$, where $v_{\text {esc }}$ and $v_{\text {Earth }}$ denote the galactic escape velocity and Earth's speed in the galaxy, respectively. We assume $v_{\text {esc }}=544 \mathrm{~km} / \mathrm{s}$ and a average $v_{\text {Earth }}=232 \mathrm{~km} / \mathrm{s}$ in subsequent calculations. As 


\begin{tabular}{ccccc}
\hline \hline & \multicolumn{2}{c}{${ }^{129} \mathrm{Xe}$} & \multicolumn{2}{c}{${ }^{131} \mathrm{Xe}$} \\
Mass $[\mathrm{GeV}]$ & $E_{R, \min }$ & $E_{R, \max }$ & $E_{R, \min }$ & $E_{R, \max }$ \\
\hline 10 & - & - & - & - \\
25 & 1.5 & 31 & - & - \\
50 & 1.2 & 110 & 6.8 & 81 \\
100 & 1.1 & 285 & 5.4 & 244 \\
250 & 1.1 & 659 & 4.9 & 601 \\
500 & 1.1 & 954 & 4.7 & 885 \\
\hline \hline
\end{tabular}

TABLE I. Minimal and maximal recoil energies, in $\mathrm{keV}$, between which inelastic scattering is allowed (see Eq. 13 ) for the two xenon isotopes and various WIMP masses.

$\frac{1}{2} \mu v_{i}^{2} \rightarrow E^{*}$, the domain of recoil energies over which the recoil spectrum is defined shrinks, converging to the value $\left(\mu v_{i}\right)^{2} / 2 m_{A}=\mu E^{*} / m_{A}$. Table I gives the minimal and maximal recoil energies for the two xenon isotopes and various WIMP masses.

We calculate the nuclear recoil spectra for elastic and inelastic, spin-dependent WIMP scattering off ${ }^{129} \mathrm{Xe}$ and ${ }^{131}$ Xe following Ref. [21]:

$$
\begin{aligned}
\frac{d R}{d E_{R}}= & \frac{\sqrt{\pi} v_{0}}{2} \frac{R_{0}}{m_{\chi} m_{A}} \frac{g\left(v_{\min }\right)}{E_{0} r} \\
& \times \frac{\sigma}{10^{-36} \mathrm{~cm}^{2}} \frac{\rho_{0}}{0.3 \mathrm{GeVcm}^{-3}} \frac{v_{0}}{220 \mathrm{~km} \mathrm{~s}^{-1}},
\end{aligned}
$$

where the WIMP-nucleus cross section $\sigma$ is given by [17:

$$
\sigma=\frac{4}{3} \frac{\pi}{2 J_{i}+1}\left(\frac{\mu}{\mu_{\text {nucleon }}}\right)^{2} S_{A}(q) \sigma_{\text {nucleon }}
$$

$R_{0}=361$ events $/(\mathrm{kgd})$ is the total event rate per unit mass for the Earth being at rest and an infinite escape velocity, $E_{0}$ is the most probable kinetic energy of an incident WIMP (given in terms of the the characteristic parameter of the Maxwell-Boltzmann distribution $\left.v_{0}\right), \rho_{0}$ is the local WIMP density in our galaxy 22, and $r$ is a kinematic factor $r=4 \mu /\left(m_{A}+m_{\chi}\right) . \quad J_{i}$ is the nuclear spin in the initial state, $\mu_{\text {nucleon }}$ is the WIMP-nucleon reduced mass, and $\sigma_{\text {nucleon }}$ is the zero momentum transfer cross-section for the nucleon. $g\left(v_{\min }\right)$ is the integral containing information about the WIMP velocity distribution $f\left(\mathbf{v}+\mathbf{v}_{\text {Earth }}\right)$ [23]:

$$
g\left(v_{\min }\right)=\int_{v_{\min }}^{\infty} \frac{f\left(\mathbf{v}+\mathbf{v}_{\text {Earth }}\right)}{v} d^{3} \mathbf{v}
$$

where the integral is from $v_{\min }$, and the velocity distribution $f\left(\mathbf{v}+\mathbf{v}_{\text {Earth }}\right)$ is truncated at $v_{\text {esc }}$.

Figure 3 shows the normalized velocity integral, $g\left(v_{\min }\right) / g(0)$, for three WIMP velocity distributions [25]: the Standard Halo Model [25], the Double Power Law profile [26], and the Tsallis model [27, 28. The different types of interactions are sensitive to different velocities. While for elastic scatters, the lowest $v_{\min }$ is determined by a detector's energy threshold, in the inelastic

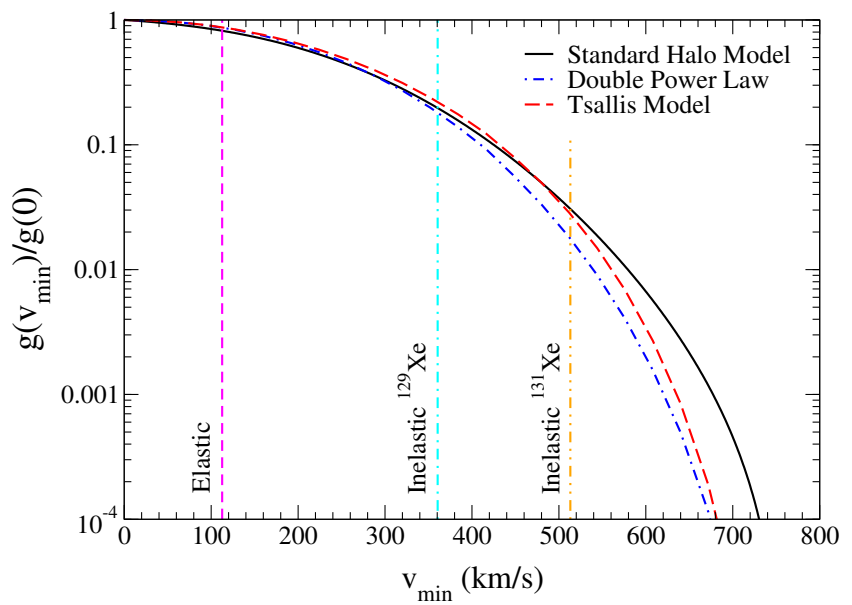

FIG. 3. (Color online) $g\left(v_{\min }\right) / g(0)$ as a function of $v_{\min }$ for several WIMP velocity distributions. The lowest values of $v_{\min }$ are shown for elastic Xe (magenta), inelastic ${ }^{129} \mathrm{Xe}$ (cyan), and inelastic ${ }^{131} \mathrm{Xe}$ (yellow) scattering. In the elastic case, $v_{\min }$ is calculated at XENON100's nuclear recoil energy threshold of $\sim 7 \mathrm{keV}$ (neglecting the difference in the nuclear mass number $A$ ) 24].

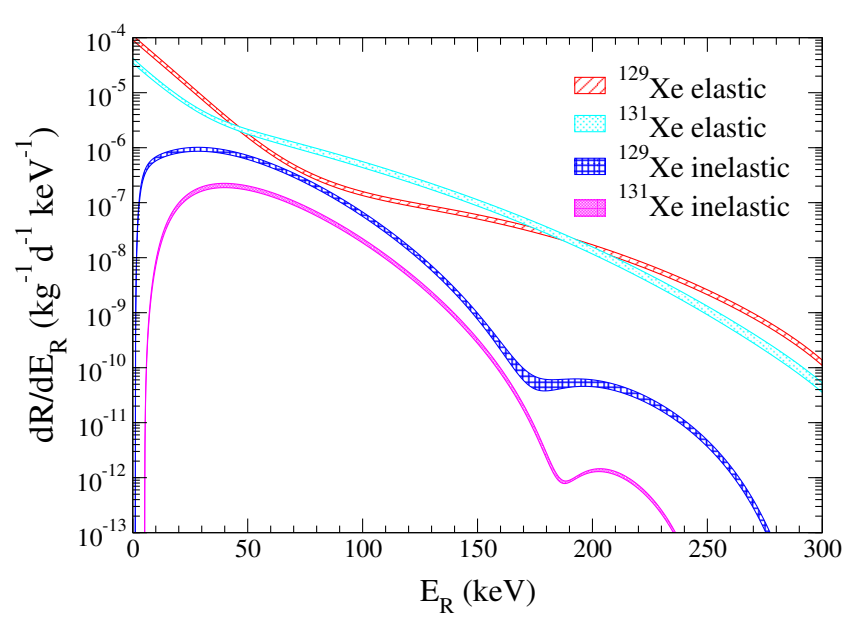

FIG. 4. (Color online) Differential nuclear recoil spectra $d R / d E_{R}$ as a function of recoil energy $E_{R}$ for scattering off ${ }^{129} \mathrm{Xe}$ and ${ }^{131} \mathrm{Xe}$, assuming "neutron-only" couplings of a $100 \mathrm{GeV}$ WIMP with a WIMP-nucleon cross section $\sigma_{\text {nucleon }}=10^{-40} \mathrm{~cm}^{2}$. Both elastic and inelastic recoil spectra are shown for comparison, including chiral $1 b+2 b$ currents, where the bands include the uncertainties due to WIMPnucleon currents.

case the lowest $v_{\min }$ occurs at $\sqrt{2 E^{*} / \mu}$ (or recoil energy $E_{R}=\mu E^{*} / m_{A}$, see the discussion above).

Figure 4 shows the differential recoil spectra for scattering off ${ }^{129} \mathrm{Xe}$ and ${ }^{131} \mathrm{Xe}$ for "neutron-only" couplings and for the structure factors that include chiral $1 b+2 b$ currents. The elastic structure factors are taken from Ref. [15. The widths of the bands reflect the theoretical uncertainties in the WIMP-nucleon currents. The elas- 
tic, spin-dependent spectra are shown for comparison. For illustration, we show all spectra here and in the following over a wide range of expected rates but note that, for the interpretation of future experimental results, only the highest-rate parts will be relevant. As expected, the inelastic spectra fall to zero at the bounds of the recoil energy, and the domain for ${ }^{131} \mathrm{Xe}$ is smaller because of the higher excitation energy compared to ${ }^{129} \mathrm{Xe}$.

\section{SIGNATURES OF INELASTIC DARK MATTER SCATTERING}

Dark matter detectors that use liquid xenon can be expected among those most sensitivite to inelastic, spindependent WIMP scattering [29]. In these detectors, the nuclear recoil and subsequent gamma emission will occur at the same space-time coordinates: the half-lives of the lowest excited states in ${ }^{129} \mathrm{Xe}$ and ${ }^{131} \mathrm{Xe}$ are $0.97 \mathrm{~ns}$ and $0.48 \mathrm{~ns}$, respectively, and the mean free paths of gammas with energies of $39.6 \mathrm{keV}$ and $80.2 \mathrm{keV}$ in liquid xenon are $\sim 0.15 \mathrm{~mm}$ and $0.92 \mathrm{~mm}$, whereas experimental resolutions are typically of order $10 \mathrm{~ns}$ and $3 \mathrm{~mm} \mathrm{30}$. Experiments can therefore search for a total energy deposition,

$$
E_{\mathrm{vis}}=f\left(E_{R}\right) \times E_{R}+E^{*},
$$

in the detector, where $E_{\text {vis }}$ is the observed energy and $f\left(E_{R}\right)$ is an energy-dependent quenching factor for nuclear recoils 21]. Only a fraction $f\left(E_{R}\right)$ of the nuclear recoil energy will be transferred to electronic excitations. These can be observed as scintillation light in a single-phase detector or as prompt scintillation and delayed charge signals in liquid xenon time projection chambers 31, 32]; the rest is transferred to heat and remains undetected.

Figure 5 shows the differential nuclear recoil spectra for inelastic scattering off ${ }^{129} \mathrm{Xe}$ and ${ }^{131} \mathrm{Xe}$, the differential energy spectra of the de-excitation gammas, and the sum of these two contributions. The energy scale is based on the total number of quanta detected in a liquid xenon dark matter experiment. For nuclear recoils, we assume the Lindhard theory 33] with a conservative choice of the proportionality constant between the electronic stopping power and the velocity of the recoiling xenon atom, $k=0.110$, as shown in Figure 1 of Ref. 34. For the gamma lines, we assume an energy resolution as obtained in the XENON100 detector at these energies in inelastic neutron-xenon scatters 30, using a linear combination of the primary scintillation and proportional scintillation signals 35: $\sigma / E=9 \%$ at $40 \mathrm{keV}$ and $\sigma / E=6.5 \%$ at $80 \mathrm{keV}$.

Due to the prompt gamma from the nuclear deexcitation, the region of interest for the dark matter search is shifted to higher energies compared to elastic scattering. Thus, experiments can search in their observed differential energy spectra for clear structures around $40 \mathrm{keV}\left({ }^{129} \mathrm{Xe}\right)$ and $80 \mathrm{keV}\left({ }^{131} \mathrm{Xe}\right)$, although the higher energy region is kinematically suppressed. This
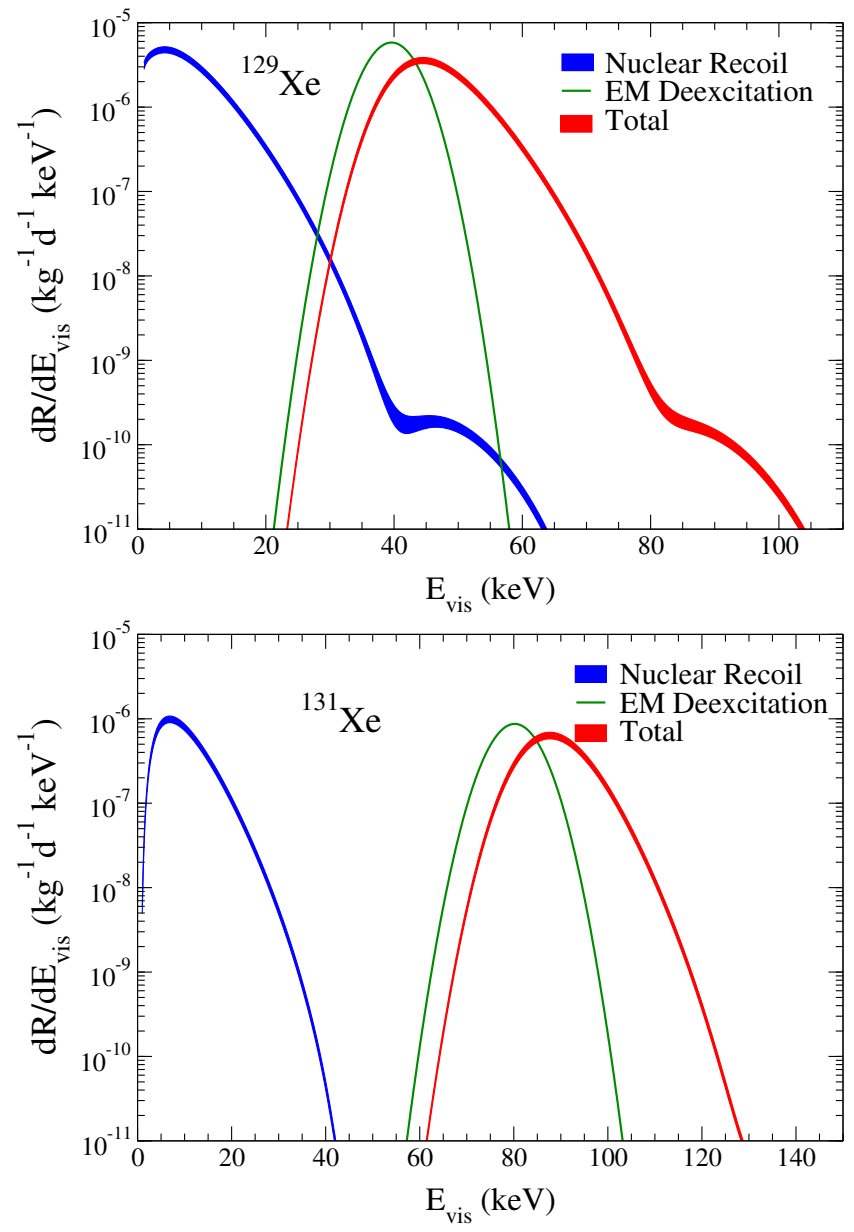

FIG. 5. (Color online) Differential energy spectra as a function of $E_{\mathrm{vis}}$ for inelastic scattering off ${ }^{129} \mathrm{Xe}$ (top) and ${ }^{131} \mathrm{Xe}$ (bottom). Shown is the nuclear recoil spectrum (blue), the de-excitation gamma (green), and their sum (red), assuming realistic detector resolution and quenching of the nuclear recoil signal. As in Figure 4, results are shown with chiral $1 b+2 b$ currents, where the bands include the uncertainties due to WIMP-nucleon currents, and we have assumed "neutron-only" couplings of a $100 \mathrm{GeV}$ WIMP with $\sigma_{\text {nucleon }}=10^{-40} \mathrm{~cm}^{2}$.

suppression is particularly pronounced for WIMP masses below $\sim 100 \mathrm{GeV}$ (see Figure 7).

In near-future detectors, observed event rates are expected to be small. While a precise measurement of the differential energy spectrum will thus be statistically prohibited, integrated rates can provide valuable insight. Figure 6 shows the integrated spectra for elastic and inelastic scattering of a $100 \mathrm{GeV}$ WIMP as well as their sum, while Figure 7 displays these for a range of WIMP masses. These spectra are shown for natural xenon, thus the isotopic contributions of each isotope, given by Eq. (14), are weighted by the aforementioned abundances. The inelastic channel is favored over the elastic one at momentum transfers above $36 \mathrm{MeV}, 45 \mathrm{MeV}$, $54 \mathrm{MeV}, 68 \mathrm{MeV}$ and $76 \mathrm{MeV}$ (corresponding to nuclear 


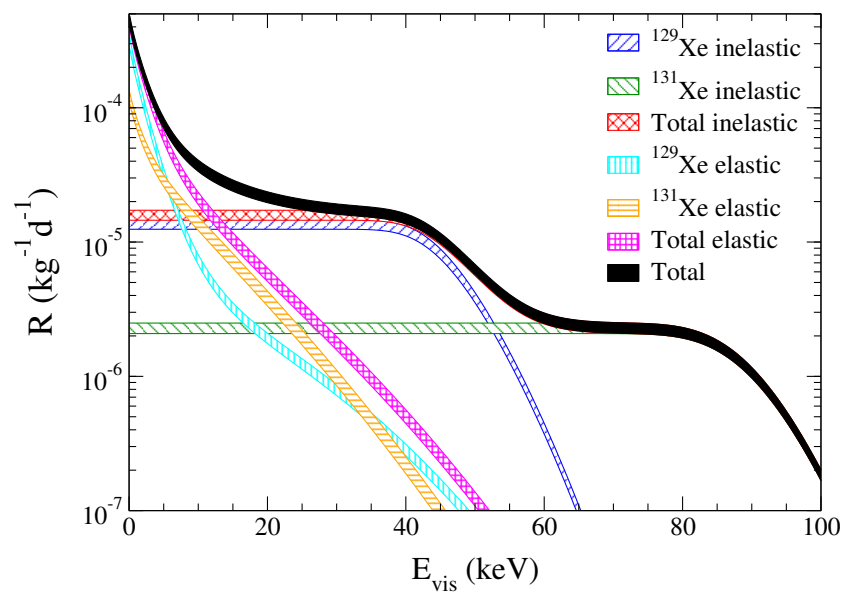

FIG. 6. (Color online) Integrated energy spectra of xenon for elastic and inelastic, spin-dependent scattering for "neutrononly" couplings and a $100 \mathrm{GeV}$ WIMP with $\sigma_{\text {nucleon }}=$ $10^{-40} \mathrm{~cm}^{2}$. The differential spectra are integrated from a given threshold value $E_{\text {vis }}$ to infinity. The inelastic contributions dominate over the elastic ones for moderate energy thresholds. As in Figure 4 results are shown with chiral $1 b+2 b$ currents, where the bands include the uncertainties due to WIMP-nucleon currents.

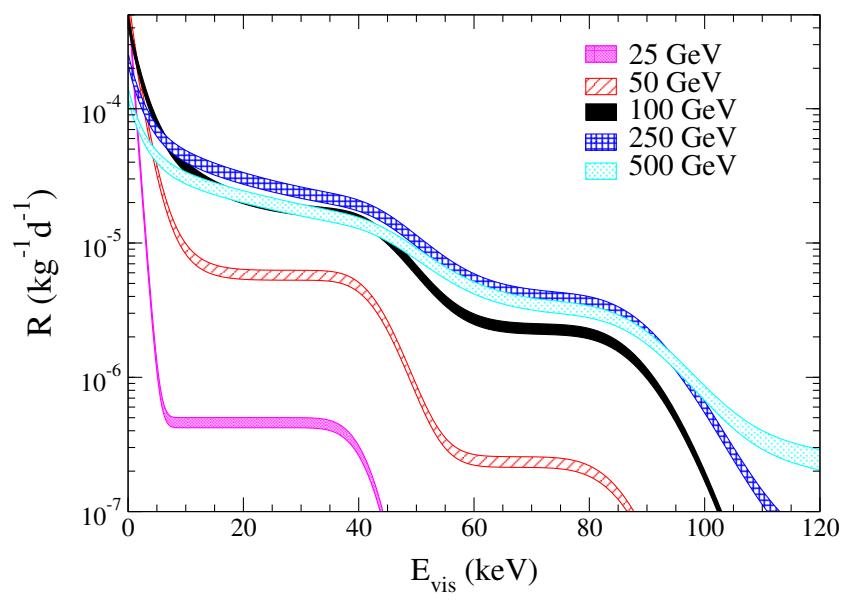

FIG. 7. (Color online) Integrated total energy spectra of xenon as in Figure 6 for various WIMP masses from $25-$ $500 \mathrm{GeV}$. The solid black curve is for a $100 \mathrm{GeV}$ WIMP as in Figure 6. In all cases, minimum energies in $E_{\text {vis }}$ exist, above which the inelastic channel dominates the elastic one. The spectrum for a $500 \mathrm{GeV}$ WIMP is lower than the for $250 \mathrm{GeV}$, because the particle number density in the galaxy decreases with increasing mass.

recoil energies of $5 \mathrm{keV}, 8 \mathrm{keV}, 12 \mathrm{keV}, 19 \mathrm{keV}$ and $24 \mathrm{keV}$ ) for WIMP masses of 25, 50, 100, 250 and $500 \mathrm{GeV}$, respectively. Should both elastic and inelastic signatures be observed by a current or future xenon experiment, it would offer a strong case for the spin-dependent nature of WIMP-nucleon interactions. On the other hand, if signal-like events are observed with no excess signal in the spin-dependent inelastic channels, this would indicate a spin-independent nature of WIMP-nucleon interactions.

\section{IMPLICATIONS FOR DARK MATTER SEARCHES AND CONCLUSIONS}

Current xenon-based dark matter experiments are either single-phase (liquid) detectors or dual-phase (liquid/gas) time projection chambers (TPCs) [31, 32. In single-phase detectors, a large liquid xenon volume is instrumented with photodetectors, and the prompt scintillation light signal is observed. In a TPC, the prompt light signal is observed with two arrays of photosensors, and, in addition, free electrons are drifted away from the interaction site toward the vapor phase above the liquid and detected via an amplified, proportional scintillation light signal. In both cases, background reduction is achieved by self-shielding and fiducialization, namely the selection of an inner, low-background liquid xenon volume based on the reconstructed vertex of each event. For TPCs, an additional tool to distinguish background from signal events is the charge-to-light ratio [36], which depends on the electronic stopping power $\mathrm{d} E / \mathrm{d} x$, and hence on the type of particle interaction.

Operational liquid xenon dark matter experiments such as XENON100 30 and XMASS 37 have reached overall background levels of $\sim 0.01$ events $/(\mathrm{kg} \mathrm{d} \mathrm{keV})$ 38 and $\sim 0.7$ events $/(\mathrm{kg} \mathrm{d} \mathrm{keV})$ 39, respectively, in the energy region $<200 \mathrm{keV}$. This implies sensitivities to inelastic, spin-dependent WIMP-nucleon cross sections of $3.8 \times 10^{-36} \mathrm{~cm}^{2}$ (XENON100) and $1.3 \times 10^{-33} \mathrm{~cm}^{2}$ (XMASS) for a WIMP with mass $130 \mathrm{GeV}$. These sensitivities are four (XENON100) and seven (XMASS) orders of magnitude worse than the current best limit for spindependent interactions from XENON100 at this WIMP mass, but could be improved by a dedicated analysis. A temporal or spatial coincidence between the nuclear recoil and the de-excitation gamma cannot be employed to further suppress the background for aforementioned reasons related to detector resolution. However, the observed background spectra are flat in this energy range, and no peak-like structures are expected from internal (such as ${ }^{222} \mathrm{Rn}$ and ${ }^{85} \mathrm{Kr}$ ) or external sources. Fast neutrons can in principle inelastically scatter off xenon nuclei, and in fact, interactions from ${ }^{241} \mathrm{AmBe}$ neutrons or similar sources are used as calibration sources for both elastic and inelastic interactions [30. However, current dark matter experiments are designed such that a background level of $\ll 1$ elastic nuclear recoil per given exposure is expected from neutrons, implying an even lower rate for inelastic neutron scatters. Hence, a dedicated analysis can search for a peak-like enhancement in an otherwise flat background spectrum. Additionally, twophase detectors can discriminate between the purely electronic recoil background and the WIMP-induced signal, which contains an additional nuclear recoil component based on the charge-to-light ratio. 


\begin{tabular}{cccc}
\hline \hline Mass $[\mathrm{GeV}]$ & ${ }^{129} \mathrm{Xe}$ & ${ }^{131} \mathrm{Xe}$ & Total \\
\hline 10 & - & - & - \\
25 & 5 & - & 5 \\
50 & 7 & 17 & 9 \\
100 & 7 & 24 & 12 \\
250 & 9 & 32 & 19 \\
500 & 11 & 35 & 24 \\
\hline \hline
\end{tabular}

TABLE II. Minimum energy $E_{\mathrm{vis}}$ in $\mathrm{keV}$ above which the observed inelastic spectrum for ${ }^{129} \mathrm{Xe},{ }^{131} \mathrm{Xe}$ and for the total spectrum starts to dominate the elastic one for various WIMP masses.

The LUX experiment [40, which started a first physics run in 2013, is expected to reach a background level that is a factor 10 below the one of XENON100. Future experiments such as XMASS-5t 41, XENON1T 42, LZ [43, and DARWIN [44, 45] are to lower their backgrounds by yet another factor of $100-1000$, compared to XENON100. This reduction can be expected to continue until the overall electronic-recoil background will be dominated by irreducible neutrinoelectron scatters from solar neutrinos at the level of $8 \times 10^{-6}$ events $/(\mathrm{kg} \mathrm{d} \mathrm{keV})$ [44, at which point further improvements in sensitivity to this interaction channel will be inhibited.

A particular experiment, using the same detector, target, and isotopic abundances, can measure both the elastic and inelastic recoil spectra. Because, for a given halo model, the ratio of the cross sections for elastic and inelastic scattering is fixed, these measured spectra would provide insight into the characteristics of the dark matter halo. For instance, the relative contributions of the elastic and inelastic scattering to the spin-dependent channel may be compared in order to inspect the WIMP velocity distribution. The fraction of the event rate that each channel contributes is necessarily connected to the integral of the velocity distribution given in Eq. (14) and shown in Figure 3, such that any deviation from the expected contributions would reflect a deviation in the expected WIMP velocity distribution.

The mass of the dark matter particle is uniquely connected to both the recoil energy domain and to the point at which the inelastic channel dominates the elastic one, thereby providing two methods by which to use the observed spectra to probe the WIMP mass. Table II lists the energies at which the inelastic spectrum starts to dominate the elastic one for the WIMP masses considered in Sect. IV] Due to the kinematic constraints discussed in Sect. III inelastic scattering is not possible for ${ }^{129} \mathrm{Xe}$ at WIMP masses below $14 \mathrm{GeV}$ or for ${ }^{131} \mathrm{Xe}$ at masses below $31 \mathrm{GeV}$.

Once a potential dark matter signal is observed in a xenon-based experiment, one could use a target enriched or depleted in ${ }^{129} \mathrm{Xe}$ and ${ }^{131} \mathrm{Xe}$. Thus, one would control the ratio of odd-even isotopes to enhance or deplete certain types of interactions. While the observed rate in the spin-independent channel would remain roughly unchanged, the rate in the spin-dependent channel, both elastic and inelastic, would increase or decrease with the fraction of odd xenon isotopes in the detector.

Inelastic scattering of dark matter can also be studied in other isotopes considered for searches of spindependent WIMP scattering. The nucleus ${ }^{73} \mathrm{Ge}$ would be especially promising [46] as it has a very low-lying state at $13 \mathrm{keV}$, plus two other low-lying excited states at 67 and $69 \mathrm{keV}$. In addition, ${ }^{127} \mathrm{I}$ [47] has a low-lying state at $58 \mathrm{keV}$. The first excited states of lighter isotopes relevant to experimental searches $\left({ }^{19} \mathrm{~F}\right.$ and $\left.{ }^{23} \mathrm{Na}\right)$ have higher excitation energies $>100 \mathrm{keV}$. Combined with the smaller reduced WIMP-nucleus mass, these lighter nuclei are therefore not sensitive to inelastic scattering.

In conclusion, we have shown that for spin-dependent WIMP-nucleus interactions, inelastic scattering dominates over elastic scattering if the momentum transfer is above a value that is still kinematically accessible for galactic dark matter to scatter off a terrestrial xenon target. To this end, we performed detailed calculations of the inelastic, spin-dependent nuclear structure factors for ${ }^{129} \mathrm{Xe}$ and ${ }^{131} \mathrm{Xe}$, which include estimates of the theoretical uncertainties in WIMP-nucleon currents. If the observed energy in a given detector is limited to a value above $4-16 \mathrm{keV}$, depending on the WIMP mass, the sensitivity to spin-dependent WIMP-nucleon interactions will be given by the inelastic channel alone. This can have important consequences for the appropriate analysis strategy. In addition, it is conceivable that detectors that are optimized for a higher energy threshold but lower radioactive background levels 48 can obtain competitive spin-dependent WIMP sensitivity using this channel alone, provided their target contains an isotope with an exited state that is kinematically accessible. Finally, this additional detection channel can provide useful information to disentangle a potential nuclear recoil signal from other nuclear recoil backgrounds. If signal events are observed in the elastic channel, simultaneous detection of signal events in the inelastic channel will point towards a spin-dependent nature of the interaction. With sufficient statistics, data from a single detector can be analyzed to extract information about the particle mass and the dark matter halo in a fully complementary way, breaking degeneracies otherwise inherent to analyses focusing on the elastic channel alone.

\section{ACKNOWLEDGEMENTS}

This work was supported by the SNF through Grant 200020-138225, the Helmholtz Association through the Helmholtz Alliance Program, contract HA216/EMMI "Extremes of Density and Temperature: Cosmic Matter in the Laboratory", the DFG through Grant SFB 634, the NSF under Grant No. 1206061, and the Purdue Research Foundation. We would like to thank the referee for very useful comments. 
[1] F. Nesti and P. Salucci, JCAP 1307, 016 (2013)

[2] J. L. Feng, Annu. Rev. Astron. Astrophys. 48, 495 (2010)

[3] G. Bertone, Particle dark matter: Observations, models and searches(Cambridge University Press, 2010)

[4] L. Baudis, Phys. Dark Univ. 1, 94 (2012)

[5] J. R. Ellis, R. A. Flores, and J. D. Lewin, Phys. Lett. B 212, 375 (1988)

[6] H. Ejiri, K. Fushimi, and H. Ohsumi, Phys. Lett. B 317, 14 (1993)

[7] P. Belli, R. Bernabei, V. Landoni, F. Montecchia, W. di Nicolantonio, A. Incicchitti, D. Prosperi, C. Bacci, and C. J. Dai, Phys. Lett. B 387, 222 (1996)

[8] F. T. Avignone III, R. L. Brodzinski, A. A. Klimenko, H. S. Miley, S. B. Osetrov, J. H. Reeves, A. A. Smolnikov, and S. I. Vasiliev, Phys. Atom. Nucl. 63, 1264 (2000)

[9] R. Bernabei et al., New J. Phys. 2, 15 (2000)

[10] J. Engel and P. Vogel, Phys. Rev. D 61, 063503 (2000)

[11] E. Caurier, G. Martínez-Pinedo, F. Nowacki, A. Poves, and A. P. Zuker, Rev. Mod. Phys. 77, 427 (2005)

[12] E. Caurier, J. Menéndez, F. Nowacki, and A. Poves, Phys. Rev. Lett. 100, 052503 (2008)

[13] J. Menéndez, A. Poves, E. Caurier, and F. Nowacki, Nucl. Phys. A 818, 139 (2009)

[14] J. Menéndez, D. Gazit, and A. Schwenk, Phys. Rev. D 86, 103511 (2012)

[15] P. Klos, J. Menéndez, D. Gazit, and A. Schwenk, Phys. Rev. D 88, 083516 (2013)

[16] P. Toivanen, M. Kortelainen, J. Suhonen, and J. Toivanen, Phys. Rev. C 79, 044302 (2009)

[17] J. Engel, S. Pittel, and P. Vogel, Int. J. Mod. Phys. E 1, 1 (1992)

[18] J. Menéndez, D. Gazit, and A. Schwenk, Phys. Rev. Lett. 107, 062501 (2011)

[19] B. Friman and A. Schwenk, Lee, S. (ed.): From Nuclei to Stars, Festschrift in Honor of Gerald E. Brown(World Scientific, 2011), arXiv:1101.4858

[20] P. Toivanen, M. Kortelainen, J. Suhonen, and J. Toivanen, Phys. Lett. B 666, 1 (2008)

[21] P. F. Smith and J. D. Lewin, Phys. Rept. 187, 203 (1990)

[22] J. Bovy and S. Tremaine, Astrophys. J. 756, 89 (2012)

[23] Y.-Y. Mao, L. E. Strigari, and R. H. Wechsler, arXiv:1304.6401

[24] E. Aprile et al. (XENON100 Collaboration), Phys. Rev. Lett. 109, 181301 (2012)

[25] M. T. Frandsen, F. Kahlhoefer, C. McCabe, S. Sarkar, and K. Schmidt-Hoberg, JCAP 1201, 024 (2012)

[26] J. F. Navarro, C. S. Frenk, and S. D. M. White, Astrophys. J. 462, 563 (1996)

[27] F. S. Ling, E. Nezri, E. Athanassoula, and R. Teyssier, JCAP 1002, 012 (2010)

[28] J. D. Vergados, S. H. Hansen, and O. Host, Phys. Rev. D 77, 023509 (2008)

[29] E. Aprile et al. (XENON100 Collaboration), Phys. Rev. Lett. 111, 021301 (2013)

[30] E. Aprile et al. (XENON100 Collaboration), Astropart. Phys. 35, 573 (2012)

[31] V. Chepel and H. Araujo, JINST 8, R04001 (2013)

[32] E. Aprile and L. Baudis, Bertone, G. (ed.): Particle dark matter: Observations, models and searches,, 413(Cambridge University Press, 2010)

[33] J. Lindhard, V. Nielsen, M. Scharff, and P. V. Thomsen, Mat. Fys. Medd. Dan. Vid. Selsk. 33, 10 (1963)

[34] P. Sorensen and C. E. Dahl, Phys. Rev. D 83, 063501 (2011)

[35] E. Aprile, K. L. Giboni, P. Majewski, K. Ni, and M. Yamashita, arXiv:0704.1118

[36] E. Aprile et al., Phys. Rev. Lett. 97, 081302 (2006)

[37] K. Abe et al., Nucl. Instrum. Meth. A 716, 78 (2013)

[38] E. Aprile et al. (XENON100 Collaboration), Phys. Rev. D 83, 082001 (2011)

[39] K. Abe et al., arXiv:1212.6153

[40] D. S. Akerib et al. (LUX Collaboration), Nucl. Instrum. Meth. A 704, 111 (2013)

[41] K. Martens for the XMASS Collaboration, 33rd International Cosmic Ray Conference, Rio de Janeiro(2013)

[42] E. Aprile for the XENON1T collaboration, arXiv:1206.6288

[43] D. C. Malling et al., arXiv:1110.0103

[44] L. Baudis for the DARWIN Consortium, J. Phys. Conf. Ser. 375, 012028 (2012)

[45] M. Schumann for the DARWIN Consortium, arXiv:1111.6251

[46] E. Holmlund, M. Kortelainen, T. S. Kosmas, J. Suhonen, and J. Toivanen, Phys. Lett. B 584, 31 (2004)

[47] J. Vergados, H. Ejiri, and K. Savvidy, Nucl. Phys. B 877, 36 (2013)

[48] M. Auger et al. (EXO Collaboration), Phys. Rev. Lett. 109, 032505 (2012) 


\section{Appendix}

TABLE III. Fits to the isoscalar/isovector structure factors $S_{00}, S_{11}$ and $S_{01}$ as well as "proton-only" and "neutron-only" structure factors $S_{p}$ and $S_{n}$ for spin-dependent WIMP elastic scattering off ${ }^{129} \mathrm{Xe}$ and ${ }^{131}$ Xe nuclei from Ref. [15], including $1 \mathrm{~b}$ and $2 \mathrm{~b}$ currents as in Fig. 2. The upper and lower limits from the theoretical error band were used for the fit. The fitting function of the dimensionless variable $u=p^{2} b^{2} / 2$ is $S_{i j}(u)=e^{-u} \sum_{n=0}^{9} c_{i j, n} u^{n}$. The rows give the coefficients $c_{i j, n}$ of the $u^{n}$ terms in the polynomial.

\begin{tabular}{|c|c|c|c|c|c|}
\hline \multicolumn{6}{|c|}{${ }^{129}$ Xe elastic } \\
\hline$e^{-u} \times$ & $S_{00}$ & $S_{11}(1 \mathrm{~b}+2 \mathrm{~b} \min )$ & $S_{11}(1 \mathrm{~b}+2 \mathrm{~b} \max )$ & $S_{01}(1 \mathrm{~b}+2 \mathrm{~b} \min )$ & $S_{01}(1 \mathrm{~b}+2 \mathrm{~b} \max )$ \\
\hline 1 & 0.0547144 & 0.0221559 & 0.0357742 & -0.0885644 & -0.0696691 \\
\hline$u$ & -0.146407 & -0.0656100 & -0.107895 & 0.254049 & 0.197380 \\
\hline$u^{2}$ & 0.180603 & 0.0863920 & 0.145055 & -0.332322 & -0.254839 \\
\hline$u^{3}$ & -0.125526 & -0.0631729 & -0.108549 & 0.244981 & 0.185896 \\
\hline$u^{4}$ & 0.0521484 & 0.0278792 & 0.0490401 & -0.109298 & -0.0825294 \\
\hline$u^{5}$ & -0.0126363 & -0.00756661 & -0.0136169 & 0.0296705 & 0.0224322 \\
\hline$u^{6}$ & 0.00176284 & 0.00126767 & 0.00233283 & -0.00492657 & -0.00375109 \\
\hline$u^{7}$ & $-1.32501 \times 10^{-4}$ & $-1.27755 \times 10^{-4}$ & $-2.39926 \times 10^{-4}$ & $4.88467 \times 10^{-4}$ & $3.77179 \times 10^{-4}$ \\
\hline$u^{8}$ & $4.23423 \times 10^{-6}$ & $7.10322 \times 10^{-6}$ & $1.35553 \times 10^{-5}$ & $-2.65022 \times 10^{-5}$ & $-2.09510 \times 10^{-5}$ \\
\hline$u^{9}$ & $-1.68052 \times 10^{-9}$ & $-1.67272 \times 10^{-7}$ & $-3.21404 \times 10^{-7}$ & $5.98909 \times 10^{-7}$ & $4.92362 \times 10^{-7}$ \\
\hline$e^{-u} \times$ & & $S_{p}(1 \mathrm{~b}+2 \mathrm{~b} \min )$ & $S_{p}(1 \mathrm{~b}+2 \mathrm{~b} \max )$ & $S_{n}(1 \mathrm{~b}+2 \mathrm{~b} \min )$ & $S_{n}(1 \mathrm{~b}+2 \mathrm{~b} \max )$ \\
\hline 1 & & 0.00196369 & 0.00715281 & 0.146535 & 0.179056 \\
\hline$u$ & & -0.00119154 & -0.0134790 & -0.409290 & -0.508334 \\
\hline$u^{2}$ & & -0.00324210 & 0.00788823 & 0.521423 & 0.657560 \\
\hline$u^{3}$ & & 0.00622602 & 0.00311153 & -0.374011 & -0.477988 \\
\hline$u^{4}$ & & -0.00496653 & -0.00653771 & 0.162155 & 0.209437 \\
\hline$u^{5}$ & & 0.00224469 & 0.00375478 & -0.0424842 & -0.0554186 \\
\hline$u^{6}$ & & $-5.74412 \times 10^{-4}$ & -0.00105558 & 0.00674911 & 0.00889251 \\
\hline$u^{7}$ & & $8.31313 \times 10^{-5}$ & $1.59440 \times 10^{-4}$ & $-6.33434 \times 10^{-4}$ & $-8.42977 \times 10^{-4}$ \\
\hline$u^{8}$ & & $-6.41114 \times 10^{-6}$ & $-1.25055 \times 10^{-5}$ & $3.20266 \times 10^{-5}$ & $4.30517 \times 10^{-5}$ \\
\hline$u^{9}$ & & $2.07744 \times 10^{-7}$ & $4.04987 \times 10^{-7}$ & $-6.54245 \times 10^{-7}$ & $-8.88774 \times 10^{-7}$ \\
\hline \multirow{2}{*}{\multicolumn{6}{|c|}{$\begin{array}{c}{ }^{131} \text { Xe elastic } \\
u=p^{2} b^{2} / 2, b=2.2905 \mathrm{fm}\end{array}$}} \\
\hline & & & & & \\
\hline$e^{-u} \times$ & $S_{00}$ & $S_{11}(1 \mathrm{~b}+2 \mathrm{~b} \min )$ & $S_{11}(1 \mathrm{~b}+2 \mathrm{~b} \max )$ & $S_{01}(1 \mathrm{~b}+2 \mathrm{~b} \min )$ & $S_{01}(1 \mathrm{~b}+2 \mathrm{~b} \max )$ \\
\hline 1 & 0.0417857 & 0.0167361 & 0.0271052 & -0.0675438 & -0.0529487 \\
\hline$u$ & -0.111132 & -0.0472853 & -0.0812985 & 0.195710 & 0.146987 \\
\hline$u^{2}$ & 0.171306 & 0.0684924 & 0.122960 & -0.306688 & -0.225003 \\
\hline$u^{3}$ & -0.132481 & -0.0514413 & -0.0940491 & 0.243678 & 0.179499 \\
\hline$u^{4}$ & 0.0630161 & 0.0237858 & 0.0439746 & -0.118395 & -0.0888278 \\
\hline$u^{5}$ & -0.0177684 & -0.00692778 & -0.0128013 & 0.0351428 & 0.0271514 \\
\hline$u^{6}$ & 0.00282192 & 0.00124370 & 0.00227407 & -0.00622577 & -0.00499280 \\
\hline$u^{7}$ & $-2.32247 \times 10^{-4}$ & $-1.31617 \times 10^{-4}$ & $-2.35642 \times 10^{-4}$ & $6.31685 \times 10^{-4}$ & $5.31148 \times 10^{-4}$ \\
\hline$u^{8}$ & $7.81471 \times 10^{-6}$ & $7.46669 \times 10^{-6}$ & $1.28691 \times 10^{-5}$ & $-3.33272 \times 10^{-5}$ & $-2.99162 \times 10^{-5}$ \\
\hline$u^{9}$ & $1.25984 \times 10^{-9}$ & $-1.73484 \times 10^{-7}$ & $-2.77011 \times 10^{-7}$ & $6.82500 \times 10^{-7}$ & $6.81902 \times 10^{-7}$ \\
\hline$e^{-u} \times$ & & $S_{p}(1 \mathrm{~b}+2 \mathrm{~b} \min )$ & $S_{p}(1 \mathrm{~b}+2 \mathrm{~b} \max )$ & $S_{n}(1 \mathrm{~b}+2 \mathrm{~b} \min )$ & $S_{n}(1 \mathrm{~b}+2 \mathrm{~b} \max )$ \\
\hline 1 & & 0.00159352 & 0.00529643 & 0.111627 & 0.136735 \\
\hline$u$ & & -0.00207344 & -0.00528808 & -0.308602 & -0.393930 \\
\hline$u^{2}$ & & 0.00567412 & -0.00627452 & 0.474842 & 0.617924 \\
\hline$u^{3}$ & & -0.00605643 & 0.0227436 & -0.375201 & -0.488443 \\
\hline$u^{4}$ & & 0.00337794 & -0.0192229 & 0.182382 & 0.234645 \\
\hline$u^{5}$ & & $-6.88135 \times 10^{-4}$ & 0.00844826 & -0.0539711 & -0.0681357 \\
\hline$u^{6}$ & & $-3.42717 \times 10^{-5}$ & -0.00212755 & 0.00944180 & 0.0116393 \\
\hline$u^{7}$ & & $3.13222 \times 10^{-5}$ & $3.03972 \times 10^{-4}$ & $-9.34456 \times 10^{-4}$ & -0.00111487 \\
\hline$u^{8}$ & & $-4.02617 \times 10^{-6}$ & $-2.27893 \times 10^{-5}$ & $4.73386 \times 10^{-5}$ & $5.34878 \times 10^{-5}$ \\
\hline$u^{9}$ & & $1.72711 \times 10^{-7}$ & $7.05661 \times 10^{-7}$ & $-9.01514 \times 10^{-7}$ & $-9.03594 \times 10^{-7}$ \\
\hline
\end{tabular}


TABLE IV. Fits to the isoscalar/isovector structure factors $S_{00}, S_{11}$ and $S_{01}$ as well as "proton-only" and "neutron-only" structure factors $S_{p}$ and $S_{n}$ for spin-dependent WIMP inelastic scattering off ${ }^{129}$ Xe nuclei, including $1 \mathrm{~b}$ and $2 \mathrm{~b}$ currents as in Fig. 1. The upper and lower limits from the theoretical error band were used for the fit. The fitting function of the dimensionless variable $u=p^{2} b^{2} / 2$ is $S_{i j}(u)=e^{-u} \sum_{n=0}^{13} c_{i j, n} u^{n}$. The rows give the coefficients $c_{i j, n}$ of the $u^{n}$ terms in the polynomial.

\begin{tabular}{|c|c|c|c|c|c|}
\hline \multicolumn{6}{|c|}{$\begin{array}{c}{ }^{129} \mathrm{Xe} \text { inelastic } \\
u=p^{2} b^{2} / 2, b=2.2853 \mathrm{fm}\end{array}$} \\
\hline$e^{-u} \times$ & $S_{00}$ & $S_{11}(1 \mathrm{~b}+2 \mathrm{~b} \min )$ & $S_{11}(1 \mathrm{~b}+2 \mathrm{~b} \max )$ & $S_{01}(1 \mathrm{~b}+2 \mathrm{~b} \min )$ & $S_{01}(1 \mathrm{~b}+2 \mathrm{~b} \max )$ \\
\hline 1 & 0.00245755 & $7.84441 \times 10^{-4}$ & 0.00126462 & -0.00352622 & -0.00277704 \\
\hline$u$ & -0.00643918 & -0.00236681 & -0.00374626 & 0.00984723 & 0.00784581 \\
\hline$u^{2}$ & 0.0402663 & 0.0178893 & 0.0257771 & -0.0637666 & -0.0538274 \\
\hline$u^{3}$ & -0.0499158 & -0.0275022 & -0.0363040 & 0.0825757 & 0.0752627 \\
\hline$u^{4}$ & 0.0268070 & 0.0203782 & 0.0228147 & -0.0449151 & -0.0495933 \\
\hline$u^{5}$ & -0.00753795 & -0.00946420 & -0.00745241 & 0.0102210 & 0.0203582 \\
\hline$u^{6}$ & 0.00116620 & 0.00318682 & 0.00101110 & $9.85679 \times 10^{-4}$ & -0.00633059 \\
\hline$u^{7}$ & $-9.84820 \times 10^{-5}$ & $-8.40630 \times 10^{-4}$ & $1.70578 \times 10^{-4}$ & -0.00142386 & 0.00168005 \\
\hline$u^{8}$ & $5.13618 \times 10^{-6}$ & $1.74690 \times 10^{-4}$ & $-1.14316 \times 10^{-4}$ & $4.91504 \times 10^{-4}$ & $-3.72182 \times 10^{-4}$ \\
\hline$u^{9}$ & $-4.08657 \times 10^{-7}$ & $-2.73828 \times 10^{-5}$ & $2.72172 \times 10^{-5}$ & $-9.94860 \times 10^{-5}$ & $6.25058 \times 10^{-5}$ \\
\hline$u^{10}$ & $4.84340 \times 10^{-8}$ & $3.04920 \times 10^{-6}$ & $-3.79530 \times 10^{-6}$ & $1.29574 \times 10^{-5}$ & $-7.32805 \times 10^{-6}$ \\
\hline$u^{11}$ & $-3.69348 \times 10^{-9}$ & $-2.24398 \times 10^{-7}$ & $3.23179 \times 10^{-7}$ & $-1.06627 \times 10^{-6}$ & $5.58160 \times 10^{-7}$ \\
\hline$u^{12}$ & $1.63951 \times 10^{-10}$ & $9.71929 \times 10^{-9}$ & $-1.55738 \times 10^{-8}$ & $5.04247 \times 10^{-8}$ & $-2.47471 \times 10^{-8}$ \\
\hline$u^{13}$ & $-3.21841 \times 10^{-12}$ & $-1.87011 \times 10^{-10}$ & $3.25850 \times 10^{-10}$ & $-1.04370 \times 10^{-9}$ & $4.84496 \times 10^{-10}$ \\
\hline$e^{-u} \times$ & & $S_{p}(1 \mathrm{~b}+2 \mathrm{~b} \min )$ & $S_{p}(1 \mathrm{~b}+2 \mathrm{~b} \max )$ & $S_{n}(1 \mathrm{~b}+2 \mathrm{~b} \min )$ & $S_{n}(1 \mathrm{~b}+2 \mathrm{~b} \max )$ \\
\hline 1 & & $1.95620 \times 10^{-4}$ & $4.65803 \times 10^{-4}$ & 0.00601911 & 0.00724841 \\
\hline$u$ & & $-3.13182 \times 10^{-4}$ & -0.00101153 & -0.0166563 & -0.0200322 \\
\hline$u^{2}$ & & 0.00203908 & 0.00478674 & 0.112020 & 0.129805 \\
\hline$u^{3}$ & & -0.00294523 & -0.00367367 & -0.152800 & -0.168826 \\
\hline$u^{4}$ & & 0.00372071 & $7.15246 \times 10^{-5}$ & 0.0969703 & 0.0946747 \\
\hline$u^{5}$ & & -0.00397760 & 0.00103907 & -0.0375392 & -0.0254257 \\
\hline$u^{6}$ & & 0.00277014 & $-6.23246 \times 10^{-4}$ & 0.0107886 & 0.00136908 \\
\hline$u^{7}$ & & -0.00122679 & $2.19242 \times 10^{-4}$ & -0.00265967 & 0.00140641 \\
\hline$u^{8}$ & & $3.56712 \times 10^{-4}$ & $-5.63446 \times 10^{-5}$ & $5.62524 \times 10^{-4}$ & $-5.71526 \times 10^{-4}$ \\
\hline$u^{9}$ & & $-6.94095 \times 10^{-5}$ & $1.05667 \times 10^{-5}$ & $-9.21422 \times 10^{-5}$ & $1.20040 \times 10^{-4}$ \\
\hline$u^{10}$ & & $8.97920 \times 10^{-6}$ & $-1.35391 \times 10^{-6}$ & $1.06409 \times 10^{-5}$ & $-1.58265 \times 10^{-5}$ \\
\hline$u^{11}$ & & $-7.41548 \times 10^{-7}$ & $1.10132 \times 10^{-7}$ & $-8.02220 \times 10^{-7}$ & $1.30826 \times 10^{-6}$ \\
\hline$u^{12}$ & & $3.53473 \times 10^{-8}$ & $-5.11319 \times 10^{-9}$ & $3.53107 \times 10^{-8}$ & $-6.19338 \times 10^{-8}$ \\
\hline$u^{13}$ & & $-7.38713 \times 10^{-10}$ & $1.03777 \times 10^{-10}$ & $-6.87368 \times 10^{-10}$ & $1.28102 \times 10^{-9}$ \\
\hline
\end{tabular}


TABLE V. Fits to the isoscalar/isovector structure factors $S_{00}, S_{11}$ and $S_{01}$ as well as "proton-only" and "neutron-only" structure factors $S_{p}$ and $S_{n}$ for spin-dependent WIMP inelastic scattering off ${ }^{131}$ Xe nuclei, including $1 \mathrm{~b}$ and $2 \mathrm{~b}$ currents as in Fig. 1. The upper and lower limits from the theoretical error band were used for the fit. The fitting function of the dimensionless variable $u=p^{2} b^{2} / 2$ is $S_{i j}(u)=e^{-u} \sum_{n=0}^{13} c_{i j, n} u^{n}$. The rows give the coefficients $c_{i j, n}$ of the $u^{n}$ terms in the polynomial.

\begin{tabular}{|c|c|c|c|c|c|}
\hline \multicolumn{6}{|c|}{$\begin{array}{c}{ }^{131} \mathrm{Xe} \text { inelastic } \\
u=p^{2} b^{2} / 2, b=2.2905 \mathrm{fm}\end{array}$} \\
\hline$e^{-u} \times$ & $S_{00}$ & $S_{11}(1 \mathrm{~b}+2 \mathrm{~b} \min )$ & $S_{11}(1 \mathrm{~b}+2 \mathrm{~b} \max )$ & $S_{01}(1 \mathrm{~b}+2 \mathrm{~b} \min )$ & $S_{01}(1 \mathrm{~b}+2 \mathrm{~b} \max )$ \\
\hline 1 & $2.35664 \times 10^{-4}$ & $1.10853 \times 10^{-4}$ & $1.79755 \times 10^{-4}$ & $-4.15211 \times 10^{-4}$ & $-3.23966 \times 10^{-4}$ \\
\hline$u$ & $-6.08853 \times 10^{-4}$ & $-2.45643 \times 10^{-4}$ & $-4.33051 \times 10^{-4}$ & 0.00129716 & $8.20866 \times 10^{-4}$ \\
\hline$u^{2}$ & 0.0755799 & 0.0334167 & 0.0530640 & -0.129644 & -0.101006 \\
\hline$u^{3}$ & -0.101995 & -0.0558344 & -0.0902236 & 0.205096 & 0.153748 \\
\hline$u^{4}$ & 0.0557595 & 0.0418290 & 0.0707274 & -0.149803 & -0.102141 \\
\hline$u^{5}$ & -0.0156606 & -0.0189589 & -0.0348228 & 0.0707352 & 0.0401662 \\
\hline$u^{6}$ & 0.00234686 & 0.00598298 & 0.0123283 & -0.0254224 & -0.0110710 \\
\hline$u^{7}$ & $-1.64837 \times 10^{-4}$ & -0.00142758 & -0.00331303 & 0.00729884 & 0.00243568 \\
\hline$u^{8}$ & $-3.56864 \times 10^{-7}$ & $2.65780 \times 10^{-4}$ & $6.73999 \times 10^{-4}$ & -0.00161524 & $-4.49005 \times 10^{-4}$ \\
\hline$u^{9}$ & $1.19267 \times 10^{-6}$ & $-3.79453 \times 10^{-5}$ & $-1.00464 \times 10^{-4}$ & $2.60489 \times 10^{-4}$ & $6.57637 \times 10^{-5}$ \\
\hline$u^{10}$ & $-1.56233 \times 10^{-7}$ & $3.95240 \times 10^{-6}$ & $1.04819 \times 10^{-5}$ & $-2.91069 \times 10^{-5}$ & $-6.99980 \times 10^{-6}$ \\
\hline$u^{11}$ & $1.31250 \times 10^{-8}$ & $-2.79009 \times 10^{-7}$ & $-7.18478 \times 10^{-7}$ & $2.12299 \times 10^{-6}$ & $4.95896 \times 10^{-7}$ \\
\hline$u^{12}$ & $-6.37159 \times 10^{-10}$ & $1.18297 \times 10^{-8}$ & $2.89173 \times 10^{-8}$ & $-9.07671 \times 10^{-8}$ & $-2.07588 \times 10^{-8}$ \\
\hline$u^{13}$ & $1.35650 \times 10^{-11}$ & $-2.26329 \times 10^{-10}$ & $-5.16254 \times 10^{-10}$ & $1.72399 \times 10^{-9}$ & $3.87968 \times 10^{-10}$ \\
\hline$e^{-u} \times$ & & $S_{p}(1 \mathrm{~b}+2 \mathrm{~b} \min )$ & $S_{p}(1 \mathrm{~b}+2 \mathrm{~b} \max )$ & $S_{n}(1 \mathrm{~b}+2 \mathrm{~b} \min )$ & $S_{n}(1 \mathrm{~b}+2 \mathrm{~b} \max )$ \\
\hline 1 & & $5.65964 \times 10^{-6}$ & $2.28585 \times 10^{-5}$ & $6.70789 \times 10^{-4}$ & $8.32428 \times 10^{-4}$ \\
\hline$u$ & & $-8.66267 \times 10^{-5}$ & $-5.09691 \times 10^{-5}$ & -0.00169273 & -0.00243141 \\
\hline$u^{2}$ & & 0.00202856 & 0.00813986 & 0.210152 & 0.258952 \\
\hline$u^{3}$ & & 0.00339658 & -0.00456893 & -0.312067 & -0.398955 \\
\hline$u^{4}$ & & -0.00915716 & -0.00374602 & 0.200540 & 0.278124 \\
\hline$u^{5}$ & & 0.00811758 & 0.00477228 & -0.0755643 & -0.122295 \\
\hline$u^{6}$ & & -0.00416169 & -0.00227425 & 0.0198702 & 0.0404361 \\
\hline$u^{7}$ & & 0.00143326 & $6.58701 \times 10^{-4}$ & -0.00421369 & -0.0108203 \\
\hline$u^{8}$ & & $-3.48016 \times 10^{-4}$ & $-1.34722 \times 10^{-4}$ & $7.63565 \times 10^{-4}$ & 0.00228304 \\
\hline$u^{9}$ & & $5.95083 \times 10^{-5}$ & $2.03323 \times 10^{-5}$ & $-1.11246 \times 10^{-4}$ & $-3.56484 \times 10^{-4}$ \\
\hline$u^{10}$ & & $-6.95756 \times 10^{-6}$ & $-2.18809 \times 10^{-6}$ & $1.18177 \times 10^{-5}$ & $3.88612 \times 10^{-5}$ \\
\hline$u^{11}$ & & $5.26485 \times 10^{-7}$ & $1.55368 \times 10^{-7}$ & $-8.36904 \times 10^{-7}$ & $-2.77670 \times 10^{-6}$ \\
\hline$u^{12}$ & & $-2.31918 \times 10^{-8}$ & $-6.46377 \times 10^{-9}$ & $3.50755 \times 10^{-8}$ & $1.16640 \times 10^{-7}$ \\
\hline$u^{13}$ & & $4.51904 \times 10^{-10}$ & $1.20019 \times 10^{-10}$ & $-6.56349 \times 10^{-10}$ & $-2.18138 \times 10^{-9}$ \\
\hline
\end{tabular}

